\title{
Heat Conduction from Donuts
}

\author{
Rajai S. Alassar and Mohammed A. Abushoshah
}

\begin{abstract}
In this paper, an exact solution of steady heat conduction from a hot donut (a torus) placed in an infinite medium of constant temperature is obtained. The governing energy equation is recast in a naturally-fit coordinates system and then solved using toroidal basis functions.
\end{abstract}

Index Terms - Donuts, heat conduction, toroidal coordinates.

\section{INTRODUCTION}

Several industries involve cooling of ring-like blanks (donuts). In such industries, which are not limited to food processing, it is very important to estimate, for example, the cooling time of such products before packaging.

The problem considered here is that of a torus (donut) with inner radius $r$ and outer radius $R$, heated to a uniform temperature $T_{s}$ and then placed in a medium of constant temperature $T_{f}$, Fig. 1. Our interest is to find the temperature distribution around the donut.

\section{Method OF SOLUTION}

A torus is generated by revolving a circle in three dimensional space about a coplanar axis which does not necessarily touch the circle. If the axis touches the circle, the resulting surface is a horn torus. Furthermore, if the axis is a chord of the circle, the resulting surface is a spindle torus. A sphere is the degenerate case when the axis is a diameter of the circle [1]- [3].

To suit the geometry of the problem, we use the Toroidal Coordinate System. The toroidal coordinate system $(\eta, \xi, \phi)$, is related to the cartesian coordinate system by the relations

$$
\begin{aligned}
& x=\frac{c \sinh \xi \cos \phi}{\cosh \xi-\cos \eta}, \quad y=\frac{c \sinh \xi \sin \phi}{\cosh \xi-\cos \eta}, \\
& z=\frac{c \sin \eta}{\cosh \xi-\cos \eta}
\end{aligned}
$$

with the corresponding scale factors given by:

$$
h_{\xi}=h_{\eta}=\frac{c}{\cosh \xi-\cos \eta}, \quad h_{\phi}=\frac{c \sinh \xi}{\cosh \xi-\cos \eta}
$$

Manuscript received October 18, 2012; revised February 27, 2013. This work was supported by King Fahd University of Petroleum \& Minerals (KFUPM) under Grant SF121 — CS-03, Saudi Arabia.

Rajai S. Alassar is with the King Fahd University, Saudi Arabia.(e-mail: alassar@kfupm.edu.sa)

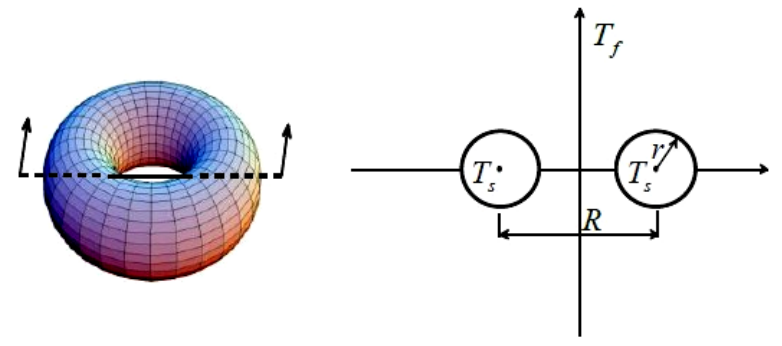

Fig. 1. Problem configuration.
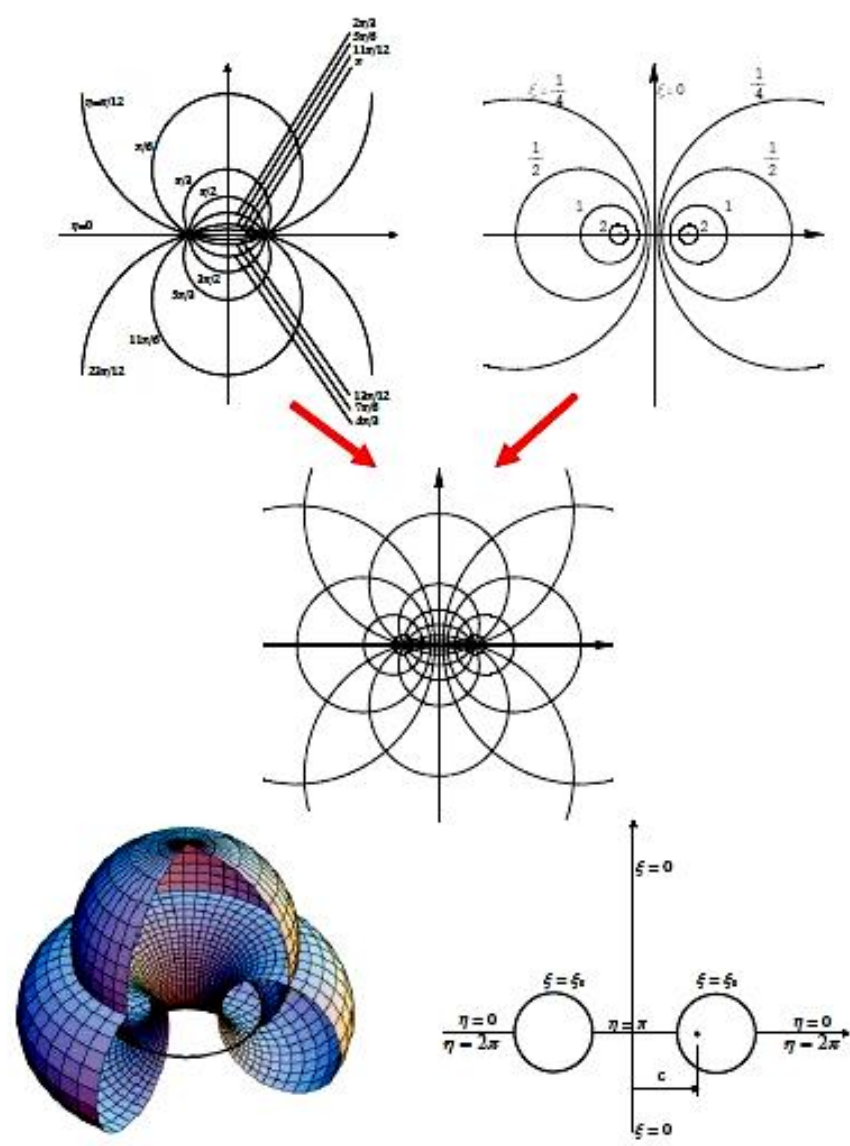

Fig. 2. Toroidal coordinates.

The coordinates satisfy $\xi \in[0, \infty), \quad \eta \in[0,2 \pi)$, and $\phi \in[0,2 \pi)$ with $c\left(c=\sqrt{R^{2}-r^{2}}\right)$ being the focal distance. The toroidal coordinate system is composed of surfaces of constant $\xi$ which are given by the toroids $x^{2}+y^{2}+z^{2}+c^{2}=2 c \sqrt{x^{2}+y^{2}}$ coth $\xi$, surfaces of constant $\eta$ given by the spherical bowls $x^{2}+y^{2}+(z-c \cot \eta)^{2}=c^{2} / \sin ^{2} \eta$, and surfaces of constant $\phi$ given by the half planes $\tan \phi=y / x$, Fig. 2 .

The steady version of the differential equation of heat conduction for a homogeneous isotropic solid with no heat 
generation is given by

$$
\nabla^{2} T=0
$$

where $T$ is the temperature, and $\alpha$ is the thermal diffusivity. In a general orthogonal curvilinear coordinates system $\left(u_{1}, u_{2}, u_{3}\right)$, equation (3) with scale factors $h_{1}, h_{2}$ and $h_{3}$ takes the form

$\frac{1}{h_{1} h_{2} h_{3}}\left[\frac{\partial}{\partial u_{1}}\left(\frac{h_{2} h_{3}}{h_{1}} \frac{\partial T}{\partial u_{1}}\right)+\frac{\partial}{\partial u_{2}}\left(\frac{h_{1} h_{3}}{h_{2}} \frac{\partial T}{\partial u_{2}}\right)+\frac{\partial}{\partial u_{3}}\left(\frac{h_{1} h_{2}}{h_{3}} \frac{\partial T}{\partial u_{3}}\right)\right]=0$

Equation (4) may be specialized for toroidal coordinates as

$$
\frac{\partial}{\partial \xi}\left(\frac{\sinh \xi}{\cosh \xi-\cos n} \frac{\partial T}{\partial \xi}\right)+\frac{\partial}{\partial \eta}\left(\frac{\sinh \xi}{\cosh \xi-\cos n} \frac{\partial T}{\partial \eta}\right)=0
$$

The boundary conditions to be satisfied are:

$$
T\left(\eta, \xi_{0}\right)=T_{s}, \quad T(0,0)=T_{f}
$$

where $\xi_{0}\left(\xi_{0}=\cosh ^{-1}\left(\frac{R}{r}\right)\right)$ defines the surface of the torus. The point $(\eta, \xi) \rightarrow(0,0)$ defines the far field away from the torus whereas the first condition in (6) indicates that that the surface of the torus is maintained at constant temperature.

We define the dimensionless temperature as

$$
u(\eta, \xi)=\frac{T-T_{f}}{T_{s}-T_{f}}
$$

Equation (5) and the boundary conditions (6) can now be rewritten in terms of the dimensionless temperature as

$$
\begin{gathered}
\frac{\partial}{\partial \xi}\left(\frac{\sinh \xi}{\cosh \xi-\cos n} \frac{\partial u}{\partial \xi}\right)+\frac{\partial}{\partial \eta}\left(\frac{\sinh \xi}{\cosh \xi-\cos n} \frac{\partial u}{\partial \eta}\right)=0 \\
u\left(\eta, \xi_{0}\right)=1, \quad u(0,0)=0
\end{gathered}
$$

Laplace equation (the energy equation (8)) is R-separable in toroidal coordinates, [4-6]. We, therefore, assume a solution of the form

$$
u(\eta, \xi) \equiv \sqrt{\cosh \xi-\cos \eta} X(\xi) Y(\eta)
$$

Upon substituting (10) in (8), and performing the necessary rituals, one finds the basis functions are $P_{n-\frac{1}{2}}(\cosh \xi), Q_{n-\frac{1}{2}}(\cosh \xi), \cos n \eta, \sin n \eta$, where $P_{n-\frac{1}{2}}$ and $Q_{n-\frac{1}{2}}$ are the toroidal functions of the first and second kinds respectively. Due to the boundary conditions in (9) and the boundedness requirements, only $P_{n-\frac{1}{2}}$ and $\cos n \eta$ survive.

The solution can then be written as

$$
u(\eta, \xi) \equiv \sqrt{\cosh \xi-\cos \eta} \sum_{n=0}^{\infty} A_{n} P_{n-\frac{1}{2}}(\cosh \xi) \cos (n \eta)
$$

We apply the first condition in (9) to get $A_{n}$. One may make use of the integral

$$
\int_{0}^{2 \pi} \frac{\cos (n \eta)}{\sqrt{\cosh \xi-\cos \eta}} d \eta=2 \sqrt{2} Q_{n-\frac{1}{2}}(\cosh \xi)
$$

to show that, [7]

$$
A_{n}=\frac{2 \sqrt{2}}{\pi\left(1+\delta_{n 0}\right)} \frac{Q_{n-\frac{1}{2}}\left(\cosh \xi_{0}\right)}{P_{n-\frac{1}{2}}\left(\cosh \xi_{0}\right)}
$$

The solution of (11), thus, is

$$
\begin{aligned}
& u(\eta, \xi) \equiv \sqrt{\cosh \xi-\cos \eta} \\
& \quad \times \sum_{n=0}^{\infty} \frac{2 \sqrt{2}}{\pi\left(1+\delta_{n 0}\right)} \frac{Q_{n-\frac{1}{2}}\left(\cosh \xi_{0}\right)}{P_{n-\frac{1}{2}}\left(\cosh \xi_{0}\right)} P_{n-\frac{1}{2}}(\cosh \xi) \cos (n \eta)
\end{aligned}
$$

where $\delta_{i j}$ is Kronecker delta.

Contour plots of the solution (14) for $r=1$ and $R=3 / 2,5 / 2$, and $7 / 2$ are shown in Figure 3 .
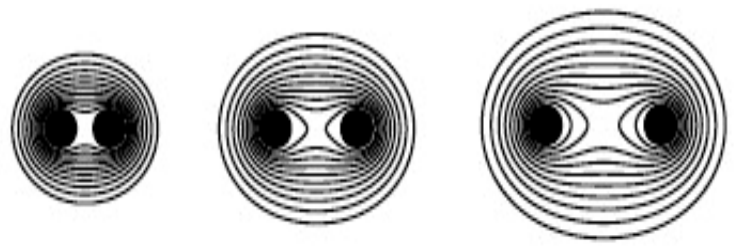

Fig. 3. Dimensionless temperature distribution for the cases $r=1$ and $R=3 / 2,5 / 2$, and $7 / 2$

\section{ACKNOWLEDGMENT}

The authors would like to thank King Fahd University of Petroleum \& Minerals (KFUPM) for supporting this research under grant FT2013-30.

\section{APPENDIX ORTHOGONAL CURVILINER COORDINATES}

A Cartesian coordinate system offers the unique advantage that all three units vectors, $\hat{\mathbf{i}}, \hat{\mathbf{j}}$, and $\hat{\mathbf{k}}$, are constant. Unfortunately, not all physical problems are well adapted to solution in Cartesian coordinates. In Cartesian coordinates we deal with three mutually perpendicular families of planes: $x=$ constant,$\quad y=$ constant, and $z=$ constant. We superimpose on this system three other families of surfaces. The surfaces of any one family need not be parallel to each other and they need not be planes. Any point is described as the intersection of three planes in Cartesian coordinates or as the intersection of the three surfaces which form curvilinear coordinates. Describing curvilinear coordinates surfaces by $u_{1}=c_{1}, u_{2}=c_{2}, u_{3}=c_{3}$ where $c_{1}, c_{2}$, and $c_{3}$ are constant we identify a point by three numbers $\left(u_{1}, u_{2}, u_{3}\right)$, called the curvilinear coordinates of the point.

Let the functional relationship between curvilinear 
coordinates $\left(u_{1}, u_{2}, u_{3}\right)$ and the Cartesian coordinates $(x, y, z)$ be given as:

$$
\begin{aligned}
& x=x\left(u_{1}, u_{2}, u_{3}\right) \\
& y=y\left(u_{1}, u_{2}, u_{3}\right) \\
& z=z\left(u_{1}, u_{2}, u_{3}\right)
\end{aligned}
$$

which can be inverted as

$$
\begin{aligned}
& u_{1}=u_{1}(x, y, z) \\
& u_{2}=u_{2}(x, y, z) \\
& u_{3}=u_{3}(x, y, z)
\end{aligned}
$$

Any point $P$ in the domain, having curvilinear coordinates $\left(c_{1}, c_{2}, c_{3}\right)$, there will pass three isotimic surfaces (on which the value of a given quantity is everywhere equal) $u_{1}(x, y, z)=c_{1}, u_{2}(x, y, z)=c_{2}$, and $u_{3}(x, y, z)=c_{3}$. As illustrated in Figure A.1, these surfaces intersect in pairs to give three curves passing through $P$, along each of which only one coordinate varies; these are the coordinates curves.

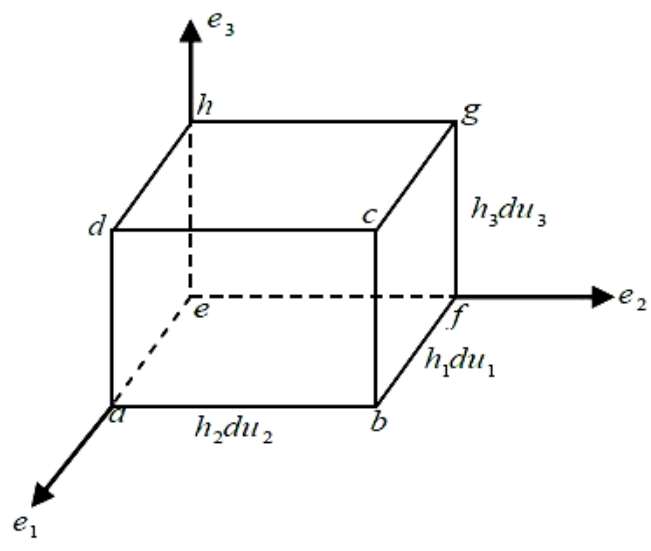

Fig. A.1. A curvilinear Coordinate System $\left(u_{1}, u_{2}, u_{3}\right)$

The normal to the surface $u_{i}=c_{i}$ is the gradient:

$$
\vec{\nabla} u_{i}=\frac{\partial u_{i}}{\partial x} \hat{\mathbf{i}}+\frac{\partial u_{i}}{\partial y} \hat{\mathbf{j}}+\frac{\partial u_{i}}{\partial z} \hat{\mathbf{k}}
$$

The tangent to the coordinate curve for $u_{i}$ is the vector

$$
\frac{\partial \vec{r}}{\partial u_{i}}=\frac{\partial x}{\partial u_{i}} \hat{\mathbf{i}}+\frac{\partial y}{\partial u_{i}} \hat{\mathbf{j}}+\frac{\partial z}{\partial u_{i}} \hat{\mathbf{k}}
$$

We say that $u_{1}, u_{2}$, and $u_{3}$ are orthogonal curvilinear coordinates whenever the vectors $\vec{\nabla} u_{1}, \vec{\nabla} u_{2}$ and $\vec{\nabla} u_{3}$ are mutually orthogonal at every point.

Each gradient vector $\vec{\nabla} u_{i}$ is parallel to the tangent vector $\frac{\partial \vec{r}}{\partial u_{i}}$ for the corresponding coordinate curve. And any coordinate curve for $u_{i}$ intersects the isotimic surface $u_{i}=c_{i}$ at right angles when $\left(u_{1}, u_{2}, u_{3}\right)$ form orthogonal curvilinear coordinate. To see this, consider a coordinate curve for $u_{1}$.

1) This curve is the intersection of two surfaces $u_{2}=c_{2}$ and $u_{3}=c_{3}$. Hence, its tangent $\frac{\partial \vec{r}}{\partial u_{1}}$ is perpendicular to both surface normals $\vec{\nabla} u_{2}$ and $\vec{\nabla} u_{3}$.

2) The vector $\vec{\nabla} u_{1}$ is also perpendicular to $\vec{\nabla} u_{2}$ and $\vec{\nabla} u_{3}$.

3) This implies that $\frac{\partial \vec{r}}{\partial u_{1}}$ is parallel to $\vec{\nabla} u_{1}$.

Since both point in the direction of increasing $u_{1}$, they are parallel. It follows, that the vectors $\frac{\partial \vec{r}}{\partial u_{1}}, \frac{\partial \vec{r}}{\partial u_{2}}$, and $\frac{\partial \vec{r}}{\partial u_{3}}$ also form a right-handed system of mutually orthogonal vectors. Define the right-handed system of mutually orthogonal unit vectors $\left(\mathbf{e}_{1}, \mathbf{e}_{2}, \mathbf{e}_{3}\right)$ by

$$
\mathbf{e}_{i}=\frac{\vec{\nabla} u_{i}}{\left|\vec{\nabla} u_{i}\right|}=\frac{\frac{\partial \vec{r}}{\partial u_{i}}}{\left|\frac{\partial \vec{r}}{\partial u_{i}}\right|} \quad i=1,2,3
$$

We need three functions $h_{i}$ known as the scale factors to express the vector operations in orthogonal curvilinear coordinates. The scale factor $h_{i}$ is defined to be the rate at which the arc length increases on the $i^{\text {th }}$ coordinate curve with respect to $u_{i}$. In other words, if $s_{i}$ denotes arc length on the $i^{\text {th }}$ coordinate curve measured in the direction of increasing $u_{i}$, then

$$
h_{i}=\frac{d s_{i}}{d u_{i}} \quad i=1,2,3
$$

Since the arc length can be expressed as

$$
d s=|d \vec{r}|=\left|\frac{\partial \vec{r}}{\partial u_{1}} d u_{1}+\frac{\partial \vec{r}}{\partial u_{2}} d u_{2}+\frac{\partial \vec{r}}{\partial u_{3}} d u_{3}\right|
$$

We see that

$$
h_{i}=\left|\frac{\partial \vec{r}}{\partial u_{i}}\right| \quad i=1,2,3
$$

Hence

$$
h_{i}^{2}=\left(\frac{\partial x}{d u_{i}}\right)^{2}+\left(\frac{\partial y}{d u_{i}}\right)^{2}+\left(\frac{\partial z}{d u_{i}}\right)^{2} \quad i=1,2,3
$$

Combine equations (A7) and (A8) shows that the displacement vector can be expressed in terms of the scale factors by

$$
d \vec{r}=h_{1} d u_{1} \mathbf{e}_{1}+h_{2} d u_{2} \mathbf{e}_{2}+h_{3} d u_{3} \mathbf{e}_{3}
$$

A differential length $d s$ in the rectangular coordinate $\operatorname{system}(x, y, z)$ is given by

$$
(d s)^{2}=(d x)^{2}+(d y)^{2}+(d z)^{2}
$$

The differential lengths $d x, d y$ and $d z$ are obtained from 
equation (A1) by differentiation

$$
\begin{aligned}
& d x=\frac{\partial x}{\partial u_{1}} d u_{1}+\frac{\partial x}{\partial u_{2}} d u_{2}+\frac{\partial x}{\partial u_{3}} d u_{3} \\
& d y=\frac{\partial y}{\partial u_{1}} d u_{1}+\frac{\partial y}{\partial u_{2}} d u_{2}+\frac{\partial y}{\partial u_{3}} d u_{3} \\
& d z=\frac{\partial z}{\partial u_{1}} d u_{1}+\frac{\partial z}{\partial u_{2}} d u_{2}+\frac{\partial z}{\partial u_{3}} d u_{3}
\end{aligned}
$$

Substitute equations (A12) into equation (A11), the differential length $d s$ in the orthogonal curvilinear coordinate system $\left(u_{1}, u_{2}, u_{3}\right)$ can be written as

$$
(d s)^{2}=d \vec{r} \bullet d \vec{r}=h_{1}^{2}\left(d u_{1}\right)^{2}+h_{2}^{2}\left(d u_{2}\right)^{2}+h_{2}^{2}\left(d u_{2}\right)^{2}
$$

In rectangular coordinates system a differential volume element $d V$ is given by

$$
d V=d x d y d z
$$

and the differential areas $d A_{x}, d A_{y}$, and $d A_{z}$ cut from the planes $x=$ constant, $y=$ constant, and $z=$ constant are given, respectively, by

$$
d A_{x}=d y d z, d A_{y}=d x d z, d A_{z}=d x d y
$$

In orthogonal curvilinear coordinates system, the elementary lengths from equation (A6) are given, by

$$
d s_{i}=h_{i} d u_{i} i=1,2,3
$$

Then, an elementary volume element $d V$ in orthogonal curvilinear coordinates system given, by

$$
d V=h_{1} h_{2} h_{3} d u_{1} d u_{2} d u_{3}
$$

The differential areas $d A_{1}, d A_{2}$ and $d A_{3}$ cut from planes $u_{1}=c_{1}, u_{2}=c_{2}$ and $u_{3}=c_{3}$ are given, respectively, by

$$
\begin{aligned}
& d A_{1}=d s_{2} d s_{3}=h_{2} h_{3} d u_{2} d u_{3} \\
& d A_{2}=d s_{1} d s_{3}=h_{1} h_{3} d u_{1} d u_{3} \\
& d A_{3}=d s_{1} d s_{2}=h_{1} h_{2} d u_{1} d u_{2}
\end{aligned}
$$

The gradient is a vector having the magnitude and direction of the maximum space rate of change. Then, for any function $\psi\left(u_{1}, u_{2}, u_{3}\right)$ the component of $\vec{\nabla} \psi\left(u_{1}, u_{2}, u_{3}\right)$ in the $\mathbf{e}_{1}$ direction is given, by

$$
\left.\vec{\nabla} \psi\right|_{1}=\frac{d \psi}{d s_{1}}=\frac{\partial \psi}{h_{1} \partial u_{1}}
$$

Since this is the rate of change of $\psi$ with respect to distance in the $\mathbf{e}_{1}$ direction. Since $\mathbf{e}_{1}, \mathbf{e}_{2}$, and $\mathbf{e}_{3}$ are mutually orthogonal unit vector, the gradient becomes

$$
\begin{aligned}
\operatorname{grad} \psi=\vec{\nabla} \psi\left(u_{1}, u_{2}, u_{3}\right) & =\frac{d \psi}{d s_{1}} \mathbf{e}_{1}+\frac{d \psi}{d s_{2}} \mathbf{e}_{2}+\frac{d \psi}{d s_{3}} \mathbf{e}_{3} \\
& =\frac{\partial \psi}{h_{1} \partial u_{1}} \mathbf{e}_{1}+\frac{\partial \psi}{h_{2} \partial u_{2}} \mathbf{e}_{2}+\frac{\partial \psi}{h_{3} \partial u_{3}} \mathbf{e}_{3}
\end{aligned}
$$

Let $\overrightarrow{\mathrm{F}}=F_{1} \mathbf{e}_{1}+F_{2} \mathbf{e}_{2}+F_{3} \mathbf{e}_{3}$ be a vector field, given in terms of the unit vectors $\mathbf{e}_{1}, \mathbf{e}_{2}$ and $\mathbf{e}_{3}$. Then the divergence of $\overrightarrow{\mathrm{F}}$, denoted $\operatorname{div} \overrightarrow{\mathrm{F}}$, or $\vec{\nabla} \bullet \overrightarrow{\mathrm{F}}$ is given, by [23]

$$
\vec{\nabla} \bullet \overrightarrow{\mathrm{F}}\left(u_{1}, u_{2}, u_{3}\right)=\lim _{\int d V \rightarrow 0} \frac{\int \overrightarrow{\mathrm{F}} \bullet d A}{\int d V}
$$

where $\int d V$ is the volume of a small region of space and $d A$ is the vector area element of this volume.

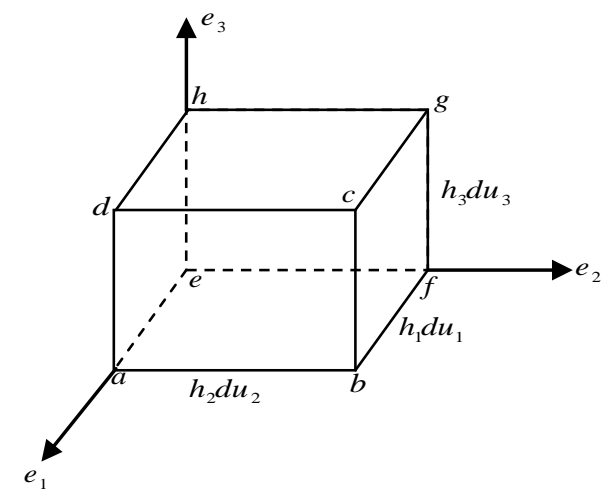

Fig. A2. Differential Rectangular Parallelepiped

We shall compute the total flux of the field $\overrightarrow{\mathrm{F}}$ outsides of the small rectangular parallelepiped Figure A2. We, then, divide this flux by the volume of the box and take the limit as the dimensions of the box go to zero. This limit is the $\vec{\nabla} \bullet \overrightarrow{\mathrm{F}}$. Note that the positive direction has been chosen so that $\left(u_{1}, u_{2}, u_{3}\right)$ or $\left(\mathbf{e}_{1}, \mathbf{e}_{2}, \mathbf{e}_{3}\right)$ from a right-hand system.

The area of the face $a b c d$ is $h_{2} h_{3} d u_{2} d u_{3}$ and the flux normal is $\overrightarrow{\mathrm{F}} \bullet \mathbf{e}_{1}=F_{1}$. Then the flux outward from the face abcd is $F_{1} h_{2} h_{3} d u_{2} d u_{3}$. The outward unit normal to the face efgh is $-\mathbf{e}_{1}$, so its outward flux is $-F_{1} h_{2} h_{3} d u_{2} d u_{3}$. Since $F_{1}, h_{2}$, and $h_{3}$ are functions of $u_{1}$ as we move along the $u_{1}$ - coordinate curve, the sum of these two is approximately

$$
\left[\frac{\partial}{\partial u_{1}}\left(F_{1} h_{2} h_{3}\right) d u_{1}\right] d u_{2} d u_{3}
$$

Adding in the similar results for the other two pairs of faces, we obtain the net flux outward from the parallelepiped is

$\left[\frac{\partial}{\partial u_{1}}\left(F_{1} h_{2} h_{3}\right)+\frac{\partial}{\partial u_{2}}\left(F_{2} h_{2} h_{3}\right)+\frac{\partial}{\partial u_{3}}\left(F_{3} h_{1} h_{2}\right)\right] d u_{1} d u_{2} d u_{3}$

Divide equation (A23) by differential volume equation (A17). Hence the flux output per unit volume is given by 


$$
\begin{aligned}
& \operatorname{div} \overrightarrow{\mathrm{F}}=\vec{\nabla} \bullet \overrightarrow{\mathrm{F}} \\
& =\frac{1}{h_{1} h_{2} h_{3}}\left[\frac{\partial}{\partial u_{1}}\left(F_{1} h_{2} h_{3}\right)+\frac{\partial}{\partial u_{2}}\left(F_{2} h_{1} h_{3}\right)+\frac{\partial}{\partial u_{3}}\left(F_{3} h_{1} h_{2}\right)\right]
\end{aligned}
$$

By using equations (A20) and (A24), the Laplacian is given as

$$
\begin{gathered}
\nabla^{2} \psi=\operatorname{div} \operatorname{grad} \psi \\
=\frac{1}{h_{1} h_{2} h_{3}}\left[\frac{\partial}{\partial u_{1}}\left(\frac{h_{2} h_{3}}{h_{1}} \frac{\partial \psi}{\partial u_{1}}\right)+\frac{\partial}{\partial u_{2}}\left(\frac{h_{1} h_{3}}{h_{2}} \frac{\partial \psi}{\partial u_{2}}\right)+\frac{\partial}{\partial u_{3}}\left(\frac{h_{1} h_{2}}{h_{3}} \frac{\partial \psi}{\partial u_{3}}\right)\right]
\end{gathered}
$$

\section{REFERENCES}

[1] P. Moon and D. E. Spencer, Field Theory for Engineers, Princeton, NJ: Van Nostrand, 1961.

[2] P. M. Morse and H. Feshbach, Methods of Theoretical Physics, Part I. New York: McGraw-Hill, 1953.

[3] G. Arfken, Mathematical Methods for Physicists, 3rd ed. Orlando, FL: Academic Press, 1985.
[4] N. N. Lebedev, Special Functions and Their Applications, Dover Publications, 1972.

[5] M. Abramowit and I. A. Stegun, Handbook of Mathematical Functions, Ninth Edition. New York: Dover, 1970.

[6] M. Andrews, "Alternative Separation of Laplace's Equation in Toroidal Coordinates and its Application to Electrostatics," Journal of Electrostatics, vol. 64, pp. 664-672, 2006.

[7] I. S. Gradshteyn and I. M. Riyzhik, Tables of Integrals, Series and Products, Fourth (English) Edition, (Prepared by Alan Jeffrey), Academic Press, New York, 1980.

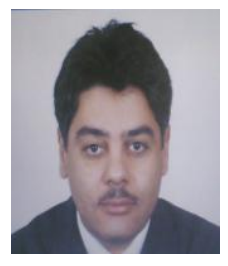

Rajai S. Alassar is a professor in the Department of Mathematics and Statistics at King Fahd University if Petroleum \& Minerals (KFUPM), Saudi Arabia. 